\title{
Interpretaciones de la medicina quiteña del siglo XVIII en torno a la curandería o mojanería
}

\section{Interpretations of Quito medicine from the 18th century around the quackery or mojanería}

DOI: https://doi.org/10.29166/tyc.v1i21.2477

\begin{abstract}
Alan Erick Rodríguez Valdivia
Cursa estudios de doctorado en Historia de los Andes en la Facultad Latinoamericana de Ciencias Sociales, FLACSO-Ecuador. Su investigación de tesis se enfoca en la interpretación de las experiencias y emociones de los padecimientos de enfermedades en contextos de brujería y curandería en la Real Audiencia de Quito desde comienzos del siglo XVIII hasta las dos primeras décadas del XIX.
\end{abstract}

Correo: geocultural@gmail.com

\section{Resumen}

El propósito de este artículo es reflexionar acerca de cómo se han silenciado las prácticas de la curandería o mojanería en algunas interpretaciones de la medicina de la Real Audiencia de Quito en el siglo XVIII. También propone algunas directrices para interpretar 0 reinterpretar esas prácticas de acuerdo con un enfoque basado en tales experiencias. Los hallazgos muestran la poca solvencia de algunas interpretaciones acerca del comportamiento de la curandería y de la complejidad de este tipo de conocimiento.

Palabras clave: Real Audiencia de Quito, historiografía de la medicina, curandería, experiencia.

\section{Abstract}

The purpose of this article is to reflect about how some practices of the healing or "mojanería" have been silenced in some interpretations of the medicine of the Royal Audience of Quito for the 18th century. As well as proposing certain guidelines for interpreting or reinterpreting these practices according to an approach based on such experiences. The findings show the little reliability of certain interpretations about the behavior of the healers, and the complexity of this type of knowledge.

Keywords: Royal Audience of Quito, historiography of medicine, healing, experience. 
El presente artículo es una reflexión acerca de cómo, hasta cierto punto, se ha silenciado a los practicantes de la curandería y la mojanería en la historiografía de la medicina quiteña ${ }^{1}$ del el Siglo XVIII y de cómo se podrían reinterpretar los comportamientos de dichos practicantes desde un enfoque basado en sus experiencias. Cuando me refiero a reflexión, quiero decir que iré mostrando datos de narraciones que, en el transcurso de mi investigación, considero importantes y también cuestionables, llevándome a precisar en algunos especialistas de la salud que, hasta el momento, han sido poco tratados o silenciados en la historiografía de la Real Audiencia de Quito.

En consecuencia, los vacíos y las interrogantes me conducen a explorar otra forma de interpretar las fuentes primarias, siendo precisamente un camino novedoso las investigaciones enmarcadas en la experiencia de la curandería o mojanería. En otras palabras, como el problema de algunas narraciones no está en el pasado que narran sino en el momento en que se dieron esas narraciones, la escritura de la historia es también una forma de comprometerse como actor y narrador (Trouillot, 2017)

He considerado oportuno reflexionar acerca de este tema debido a la reducida información que hay respecto del conocimiento de quienes fueron llamados curanderos, brujas, hechiceras o mojanes $^{2}$. Aunque se los nombra como un grupo que fue importante al momento de atender a las personas que padecían enfermedades en el siglo XVIII, sobre todo en los pueblos, es poco lo que sabemos de sus prácticas, experiencias, transformaciones e interacciones de sus conocimientos. Igualmente, me sorprende el modo en que se hacen afirmaciones acerca de los comportamientos de estos actores con muy pocas fuentes primarias que lo demuestren. Por tal motivo, en ocasiones cuestionaré ciertas narraciones de algunas fuentes que he localizado en archivo.

En cuanto al contenido, la primera sección pone su atención en los elementos estructurales que han influido en el desarrollo de la medicina en la Sierra y la Costa. Es especialmente, se ocupa de cómo las y los autores juzgan el papel de algunas instituciones que tuvieron a su cargo la salud pública, así como de la labor y el conocimiento de practicantes sin licencia en la curación de enfermedades. La segunda sección saca a relucir una serie de datos entregados por la historiografía referentes a practicantes que curaban en pueblos y que, generalmente, estuvieron etiquetados como brujos o hechiceros. Mientras que en la tercera sección recojo algunos resultados de la Historia Global de la Medicina, focalizada en los espacios del Caribe, Brasil y el Atlántico, que interpretan la experiencia, transformación y creación del conocimiento, principalmente, de la población africana en América. Finalmente, reuniré las tres temáticas tratadas para identificar qué es lo que falta, según el enfoque que he tomado, así como lo que sugieren los trabajos que en la última década se enmarcan en la interpretación de la experiencia.

\footnotetext{
Cuando me refiero a medicina quiteña hago alusión al espacio de la Real Audiencia de Quito. Cuando quiera especificar un centro poblado lo haré como ciudad de Quito.

2 Moján fue otra forma que se utilizó en la Audiencia de Quito para nombrar a un brujo o bruja. En la tercera sección del manuscrito se podrá entender que mohán fue el nombre de la categoría que usó Gómez (2017) para referirse a las y los practicantes que estudió.
} 


\section{Aspectos generales en la Sierra y la Costa}

Basados en los registros del Cabildo de Quito, Suzanne Alchon (1996) ha interpretado que en el siglo XVIII la ciudad de Quito fue considerada un lugar insalubre y de pésima higiene pública. Es así que las autoridades locales y médicas asumieron un papel activo para solucionar estos problemas. Esto se ha visto reflejado en las fuentes por el aumento en veinte veces de los acápites sobre cuestiones relacionadas con la salud pública en los registros de Cabildo, según sostiene esta autora.

Alchon agrega que el gobierno borbónico promovió la expansión del conocimiento haciendo circular nuevos textos médicos, como las obras de Borhaave, Lister, Malpigi y otros. En la Sierra, fue principalmente la élite de Quito la que pudo acceder a este conocimiento, pero sin mejorar la salud pública. A tal punto que la autora sostiene que hacia finales del siglo XVIII la Audiencia no contaba con un protomédico permanente. Esto es discutible debido a que en el Archivo Nacional del Ecuador (ANE) he hallado juicios llevados adelante por el protomédico Bernardo Delgado ${ }^{3}$ contra curanderos en la segunda mitad del siglo XVIII, en donde se especifica que él estuvo a cargo del protomedicato en Quito desde la década de 1770 hasta comienzos del siglo XIX. Con esto quiero apuntar a que, más allá de que los libros de cabildos y otras fuentes provenientes -por ejemplo, de hospitales- puedan facilitar una perspectiva amplia de la medicina en el siglo XVIII, es necesario acudir a otras fuentes

3 Corte Suprema, Sección Criminales, Caja 167, Expediente 12. -como los juicios- para entender las continuidades, los conflictos y las prácticas que se daban en este campo.

Para el caso de Guayaquil, Michael Hamerly (1973) la describe como una ciudad que sufre de mala salud, con una dieta inadecuada, malas viviendas, servicios limitados y poca educación. Solamente los hacendados, comerciantes y profesionales suplían sus necesidades. Es por esto que los acomodados gozaban de atención médica licenciada mientras que las mujeres de clase baja debían acudir a parteras y sus esposos e hijos a curanderos y barberos. Además, aunque el intermitente Hospital de la Caridad del puerto ayudaba a los pobres, en el mejor de los casos tenía pocas camas y muy escaso personal. Hamerly agrega que no es hasta comienzos del siglo XX que los médicos licenciados ingresan a los barrios Astillero y El Bajo, notorios por su insalubridad. Por ello, no resultaría nada extraño que dichos espacios fueran frecuentados por curanderos y/o barberos. La mala calidad de la salud es atribuida por este autor a la acción de los boticarios y empíricos, puesto que estos habrían contribuido a los estragos de muchas enfermedades producto del dispendio de medicinas incorrectas, con o sin receta. En otras palabras, Hamerly termina culpando a curanderos y empíricos por las enfermedades acontecidas y resaltando la importancia de la medicina licenciada.

Ampliando la escala hacia la Sierra y Costa, Eduardo Estrella (1980) sostiene que hacia finales siglo XVIII la economía de la audiencia cristalizó su condición agroexportadora, especialmente con la comercialización del cacao. Así, la Costa 
estaba en el centro hegemónico de la economía, con lo cual se da una importante migración india de la Sierra a la Costa. En lo que respecta a la salud de las personas, esto provocó que la población migrante fuera atacada por enfermedades tropicales y respiratorias, como el paludismo, a lo que se contrapone que la población costeña habría generado con anterioridad alguna forma de resistencia, mas no la serrana.

La población india de la Sierra no se recuperó tan rápidamente, como sí ocurrió en el siglo XVII (Alchon, 1996; Powers, 1994). Para Alchon, las diversas epidemias y desastres dificultaron que se incrementara la población, lo que también se relaciona con la depresión económica. Esta afirmación puede ser debatida ya que la disminución de la población serrana, así mismo, pudo deberse a la migración hacia la Costa (Aráuz, 1999).

Visto lo ocurrido en la Sierra y la Costa, en lo que sí concuerdo con la historiografía de la medicina colonial en la Audiencia es que las personas recurrieron, producto de la credibilidad y el menor costo económico de sus prácticas curativas, a diferentes practicantes de la salud (Alchon, 1996; Estrella, 1980; Hamerly, 1973; Tapia, 2018) ${ }^{4}$. Empero, no considero adecuado juzgar y generalizar a las otras prácticas médicas como incorrectas, debido a que falta estudiar, por ejemplo, si la labor de la curandería puede haber sido relevante y, muchas veces, eficaz para tratar las enfermedades de las personas en ciudades y pueblos. Principalmente en estos últimos lugares, donde aún falta por averiguar si los médicos licenciados no llegaban curar. Asimismo, es probable que la proliferación de enfermedades no se debiera únicamente a desastres naturales y epidemias, sino que se debe tener en cuenta las condiciones sociales y económicas que posibilitaron dicha proliferación.

Para terminar esta sección, me parece problemático el uso de categorías y dicotomías que tienden a generar fronteras rígidas en el conocimiento médico, algo que la reciente historiografía de la medicina ha cuestionado (Crawford, 2016; Few, 2015; Gómez, 2017; Ramírez, 2018; Schiebinger, 2017). En primer lugar, tanto Estrella $(1980,2004)$ como Alchon (1996) construyen interpretaciones en donde la medicina nativa o aborigen se distancia de la medicina científica. La medicina aborigen se replegaría a las haciendas y pueblos aledaños y en ella solamente influiría la religión católica. Mientras que la medicina científica se sustentaría en explicaciones racionales. A esto se sumaría la medicina popular que destacaba por su cercanía con la medicina religiosa y científica, resaltando la experiencia de los sangradores y barberos. Esto es discutible debido a que Crawford y el mismo Estrella han demostrado que las fronteras de la medicina entre, por ejemplo, curanderos e ilustrados fue difusa dado que aprehendían mutuamente. Además, como se registra en fuentes de la época ${ }^{5}$, los curanderos no se replegaban a los pueblos, pues migraban y aprehendían nuevos conocimientos.

4 La historiografía de la medicina ha dejado en evidencia la confluencia de prácticas en el siglo XVIII y comienzos del XIX, en donde se acumularon varias formas de conocimiento y tratamientos de enfermedades (Armus, 2002; Gänger, 2016; Warren, 2009, 2013).

5 Tanto en las acusaciones contra falsos médicos como hacia brujos, es frecuente leer que a muchos de ellos se les acusaba de forasteros que se movían por ciudades y pueblos. Sin embargo, las acusaciones de los protomédicos son más exactas sobre la presencia de curanderos en Quito y Guayaquil. ANE, Fondo Corte Suprema, Serie Civiles y Serie Criminales. 


\section{Curandería en los pueblos}

Producto de los desastres de la década de 1690, la escasez de mano de obra de la primera mitad del siglo XVIII provocó que obrajeros y terratenientes aplicaran una mayor explotación sobre la población india (Alchon, 1996). Además, el crecimiento de la población mestiza socavó la sociedad india, a lo que habría que sumar la población afrodescendiente (Tardieu, 2006), por residir en o cerca de estas comunidades. A esto se agrega un incremento de la población forastera que provocó tensiones y conflictos intracomunales por la distribución de los escasos recursos económicos y así como del poder político.

En ese contexto, Alchon (1996) propone que la 'sociedad nativa' preservó su unidad e identidad por medio de la práctica de sus creencias y ceremonias religiosas, donde se incluyen los 'rituales curativos'. La sobrevivencia de estas prácticas, de acuerdo a la autora, también se debió a la ubicación periférica que tuvo la Audiencia tanto en el Virreinato del Perú como en el de Nueva Granada, por lo que la extirpación de idolatrías -habría que sumar la Inquisición para la criminalización de prácticas idolátricas no indias- no ejerció poder sobre este territorio (Alchon, 1995, 1996; Espinosa, 2014). Así, sostiene que estas tradiciones se mantuvieron más fortalecidas que en otras áreas del Perú, el Alto Perú, o el Caribe donde actuaba también la Inquisición. Esto también es debatible, puesto que, rastreando el caso que interpreta Frank Salomon

6 ANE, Corte Suprema, Serie Indígenas, Caja 43, Expediente 7.
(1985) acerca de Lorenso Buesaquillo, de la ciudad de Pasto en $1730^{6}$, puede notarse que la Inquisición de Lima sí pudo tener injerencia sobre la Audiencia. Por ello se debe tener cuidado antes de hacer tales aseveraciones sin contar con un número suficiente de fuentes que lo puedan verificar.

Igualmente, me parece cuestionable el uso de términos descontextualizados en referencia al momento histórico que se estudia. Tanto Salomon (1985, 1996) como Alchon (1996) utilizan el término shaman para referirse a quienes fueron acusados de brujos o hechiceros. Esto puede llevar a errores de interpretación debido a que seguramente el termino shaman fue introducido por los estudios antropológicos en el siglo $\mathrm{XX}$, pero de ninguna manera hace referencia a quienes en ese momento fueron nombrados como yerbateros o mojanes.

Regresando a las cuestiones económicas y sociales, una de estas se vio reflejada en el caso de Andrés Arévalo (Salomon, 1985, 1996), quien fue juzgado por brujería en la Villa de Zaruma en 1705. Este caso registra muy bien la agresión mágica, descubriendo lo temido que fue este personaje en la comunidad del Pueblo de Paccha. Según Salomon, el contexto que habría desencadenado este juicio es el de los intereses locales hortícolas y extranjeros ganaderos. De estos últimos, Andrés manifestó ser su enemigo declarado debido a la deforestación que producían para que aparecieran ranchos ganaderos. Las agresiones de Arevalo iban desde rituales en los que masticaba espingo y tabaco, pasando por los entie- 
rros de envoltorios hasta lanzar dardos envenenados para que sus víctimas enfermaran o murieran. Al parecer, el juicio tuvo unos fines económicos de por medio, debido a que fueron familias ganaderas y también consanguíneos del supuesto brujo quienes lo iniciaron. Inclusive, apareció un hechicero o curandero que acusó a Arevalo de hacer enfermar a la gente y al ganado.

Otra causa de brujería llevada a cabo en la segunda mitad del siglo XVIII fue en la Punta de Santa Elena, donde se ha demostrado que no hubo médicos licenciados que pudieran curar a la población, razón por la cual los curanderos cumplieron una importante función ${ }^{7}$ (Laviana, 1989, 1996). La mayoría de los procesados no negó ser curandero o curandera, pero sí se negó a decir quién fue su maestra o maestro. Solamente tres personas admitieron haber aprendido de sus padres o madres: uno, de los indios colorados; otro, de los indios de Ojiva (zona de tránsito hacia la sierra de Guaranda), y un último, mirando los remedios que hacían los médicos de Guayaquil.

En lo que se refiere a la curación de enfermedades, María Laviana (1996) ha podido reconocer que la curandería aplicaba curas contra calenturas, tabardillo, sarna, mal de madre, picaduras de culebra y vista dañada, fueran o no causadas por hechicería. Era la base de su conocimiento las propiedades curativas de las plantas. Preparaban la medicina en su hogar, colocando "mesa y mantel con las yerbas e ingredientes y el vaso o mate de aguardiente, e invocando al demonio para tener acierto" ( p. 50). Los mismos ingredientes que les servían para curar enfermedades les ayudaban a romper maleficios, algunas veces enterrándolos. Por ello, su rango de acción iba desde curar el cuerpo hasta sanar el alma de sus pacientes. En otros casos, curaban invocando a Dios, o primero llamaban a Dios y la Virgen Santísima y después al diablo.

Se puede notar que la curandería poseía una estrecha relación con el uso de hierbas medicinales. Por ello, fue característico de estos practicantes saber varias propiedades y usos de diferentes hierbas que no solamente se encontraban en la Audiencia, sino que fueron compartidas por curanderos o curanderas de otras regiones (Crawford, 2016).

La quina es un buen ejemplo de cómo circulaba el conocimiento. Entendida como artefacto cultural, la quina fue evaluada y utilizada por curanderos en los Andes con sus propios métodos, mientras que comerciantes de los puertos atlánticos, como farmacéuticos y naturalistas europeos, tuvieron sus propios métodos para identificar y evaluar ese mismo árbol (Crawford, 2016). Esto conecta las redes de saber local con las del comercio y el imperio, aunque dichas redes fueron disputadas por la tenacidad de los recolectores de corteza, curanderos, comerciantes y funcionarios locales que trataban de afirmar su propio conocimiento y experiencia sobre una aparente impotencia de farmacéuticos y botánicos españoles agentes del imperio, colocando de esta forma en duda la eficacia de la ciencia europea. Con todo, el estudio de Crawford demuestra que la

7 Martha Few (2015) describe cómo en la Audiencia de Guatemala la falta de médicos y fármacos para tratar enfermos en el campo generó problemas para que los funcionarios coloniales hicieran frente a las epidemias. Con la inoculación de la viruela a partir de 1780, religiosos, médicos y mujeres y hombres indígenas y mestizos trabajaron colectivamente durante las epidemias. 
ciencia europea moderna no sólo estaba lejos de la hegemonía, sino que a veces fue impotente frente a los heterogéneos mundos sociales, culturales y naturales que conformaban el imperio.

Frente a estas disputas por el conocimiento, no se puede negar que la quina fue un medicamente del mundo andino. El ejemplo más claro fue que, hacia mediados del siglo XVIII, Charles Marie de La Condamine recurrió al curandero lojano Fernando de La Vega para poder comprender los usos y propiedades de la quina (Crawford, 2016; Estrella, 1995). No obstante, la ironía de esto se debió a que La Condamine marginó y omitió las contribuciones de sus contactos locales para llevarse el crédito del nuevo conocimiento que llevaba a Europa. A partir de este encuentro significativo de ambos conocimientos, Crawford sostiene que el conocimiento y práctica médica de los curanderos en los Andes, al igual que los naturalista europeos, fue representante de una tradición dinámica de conocimiento especializada sobre el mundo natural, por lo cual nos invita a no reducir los conocimientos indígenas a un simple conjunto de observaciones empíricas, sino a pensar en un conjunto de conocimientos tan complejos como el de las ciencias y medicina moderna europeas. Por tanto, es difícil afirmar una superioridad del conocimiento europeo e ilustrado sobre el árbol de quina y su corteza, ya que habrían sido los curanderos andinos los primeros en reconocer la eficacia de la quina para el tratamiento de las fiebres.

\section{Una historia de la experiencia}

Por lo antes visto, a excepción de Crawford (2016) y Estrella (1995), la historiografía de la medicina no ha profundizado en las transformaciones, desarrollo y/o creación de conocimiento que la población amerindia y, especialmente, africana y afrodescendiente pudieron tener en la Audiencia durante el siglo XVIII. Adam Warren (2009) ha hecho notar cómo durante el siglo XVIII en el Perú no hubo límites claros en la aplicación de la medicina, sino más bien usos paralelos. Esto demostraría los múltiples procesos de apropiación y transformación en la sociedad colonial. Asimismo, argumentó que las formas de curar entre las tradiciones españolas e indias variaron entre las poblaciones de ciudades como Lima y los pueblos de la Sierra.

En ese sentido, son relevantes los últimos estudios de la Historia Global de la Medicina, pues han demostrado cómo en el Caribe Negro (Gómez, 2013, 2017), Brasil (Sweet, 2009, 2011) y el Atlántico (Schiebinger, 2017) el entorno natural fue reinterpretado por la población africana para crear nuevas curas, así como su aprendizaje de prácticas cristianas e indígenas para adecuarlas a sus tratamientos y a los propios conocimientos sobre el cuerpo. Aunque para el caso de la Audiencia de Quito puede ser muy difícil acceder a fuentes inquisitoriales o de otro tipo referidas a la población africana como las que han utilizado Pablo Gómez, James Sweet y Londa Schiebinger en el estudio de la experiencia africana en el Caribe y el Atlántico, sus trabajos son alentadores al momento de plantear nuevas interrogantes y enfoques en el campo.

En primer lugar, Gómez (2017) plantea que los practicantes de rituales negros del Caribe en el siglo XVII crearon conocimiento bajo novedosas estrategias para ejercer poder sobre el mundo natu- 
ral por medio de prácticas sensoriales con las que demostraron su capacidad para comprender, clasificar y manipular el mundo natural. El éxito de estos practicantes los convirtió en los líderes intelectuales de una región saturada de ideas de todo el mundo. Fue a través de esto que los practicantes reinventaron continuamente la experiencia caribeña, basándose en lecturas que derivaron de los diferentes encuentros con diversas comunidades de la región. De acuerdo a esto, podríamos preguntarnos si ċacaso las prácticas sensoriales para controlar el mundo natural no fueron también utilizadas por curanderos en la Audiencia de Quito? O si, ¿su experiencia también derivó de diferentes encuentros?

Otra cuestión que considero relevante en el trabajo de Gómez (2017) es cómo toma una postura en cuanto a la narración histórica, porque decide no referirse a sus actores históricos como brujas o hechiceros pues cree que estos términos representan el mismo lenguaje de sus contemporáneos para condenar las formas negras de conocer. Véase que, al momento de buscar otro término o categoría para estudiar a los practicantes, hay una intención diferente al uso de shaman de Salomon $(1985,1996)$ y Alchon (1996) que jamás se lo cuestionan. Elige el término mohán por ser de origen amerindio, registrado por primera vez en los siglos XVI y XVII en el norte del Reino de Nueva Granada. Otras veces se refiere a estos actores como especialistas en rituales, practicantes de rituales y especialistas en salud. En algunos casos judiciales encontrados en mi investigación, he podido reconocer que a las personas acusadas de brujería las y los testigos las nombraban como moján o moan. O en el propio Virreinato de Nueva Granada, en el siglo XVIII, parece ser recurrente nombrar a practicantes como mojanes (Ariza, 2015). Sin duda, Gómez nos lleva a cuestionarnos la propia forma de nombrar a nuestros actores.

Después de haber examinado varios casos inquisitoriales, Gómez (2017) ha afirmado que el éxito de los practicantes africanos y afrodescendientes llamó la atención de los inquisidores cuando sus competidores médicos y otros curanderos los denunciaron. El asombroso mundo creado por los practicantes rituales y el éxito que tuvieron les hicieron competir con otras entidades establecidas y patrocinadas por el estado como el Protomedicato, la Iglesia Católica y otros gremios intelectuales. Como aparece en la sección de este artículo, algo similar ha reconocido la historiografía quiteña, pero sin detallar tales competencias y menos las transformaciones del conocimiento a partir de las experiencias.

Por ejemplo, Swett (2011) ha estudiado el caso del sanador africano Domingos Álvares, interpretando cómo éste desempeñó cruciales roles sociales y políticos en la sociedad esclavista brasileña del siglo XVIII. Ensambló relaciones sociales, extrajo amplios significados políticos desde los padecimientos, impartiendo estos significados a sus pacientes, constituyendo nuevas comunidades alrededor de sus ideas colectivas. Inclusive, cuando fue bautizado al catolicismo, asimiló dichos nuevos conocimientos en las estructuras y significados del voodun, pues creía que los sacerdotes católicos poseían un nuevo y poderoso voodun, lo que a él le ofrecía una nueva posibilidad de sanar a sus pacientes. La transformación y creación del conocimiento africano de sus prácticas rituales se debió a cómo se reu- 
nieron creencias portuguesas e indias con las suyas. Fue así que los africanos utilizaban rituales católicos en sus ceremonias $\mathrm{u}$ objetos como los crucifijos. El que hayan adoptado estas nuevas formas les ayudó a acomodar nuevas formas sociales y políticas, sin olvidar el simbolismo ritual y las prácticas de su tierra en África. Someterse a las curaciones de Domingos demuestra cómo los brasileños se implicaron profundamente de todos los contextos de curación.

Los diferentes contextos de curación también son avalados por Gómez (2017), ya que los modelos africanos del Caribe sobre el funcionamiento del cuerpo y el tratamiento de las enfermedades fueron ampliamente aceptados. La aceptación del conocimiento empírico de los practicantes africanos fue impulsada por los buenos resultados obtenidos al curar, debido a cómo los practicantes comenzaron a explorar los bosques, sabanas y costas de la región en busca de elementos para luchar contra una variedad de enfermedades. Además, la exploración del mundo natural fue acompañada por una lectura espiritual del reino social en el que funcionaban estas medicinas. Por todo esto, Gómez (2017) cuestiona firmemente que se puedan reducir las epistemologías de los practicantes africanos - lo que también podríamos pensar para la Audienciaúnicamente a lo que sus contemporáneos llamaban hechicería o brujería. Esto conduce a tratar de interpretar en los contextos sociales cómo se produce la eficacia de las curas de quienes practican la medicina, en donde se podrían integrar complementos analíticos como la eficacia simbólica (Lévi-Strauss, 1995).
Gómez (2017) va más allá para argumentar que la experiencia caribeña formó la base para la creación de la autoridad en torno a los asuntos corporales. A raíz de eso, las categorías europeas asociadas a esta epistemología, como brujería y hechicería, fueron usadas como armas para ejercer control social sobre el conocimiento del cuerpo.

Por último, el conocimiento sobre el cuerpo también ha sido estudiado por Schiebinger (2017) en lo que denomina una cultura experimental en el mundo Atlántico. Esta autora introduce preguntas importantes sobre cómo las curas africanas fueron transportadas a América, o si los remedios amerindios fueron transmitidos a los africanos, o inclusive si los esclavos desarrollaron curas en las plantaciones. Como afirma, todavía para el siglo XVIII en el mundo Atlántico hubo una fuerte mezcla y competencia entre las enfermedades amerindias, africanas y europeas, los medicamentos y los practicantes. Por lo mismo, los europeos, desde el siglo XVI hasta el XVIII, tendieron a valorar los conocimientos médicos de los pueblos con los que se encontraron en todo el mundo.

Médicos y curanderos de todo tipo en las Indias Occidentales sirvieron como intermediarios del conocimiento, seleccionando trozos y piezas valiosas de estas tradiciones para crear curas ocasionalmente efectivas (Schiebinger, 2017). Pero cuando los esclavos tenían una cura efectiva, a menudo los europeos insistían en probarla con sus propios métodos. Siendo así que las curas, africanas o amerindias, fueron probadas por los establecimientos médicos europeos de acuerdo con sus procedimientos. 


\section{Conclusión}

La situación sanitaria en la Audiencia de Quito durante el siglo XVIII no gozó de prosperidad dadas las condiciones negativas que ha argumentado la historiografía. Bajo esas condiciones, inclusive en las ciudades fue escasa la acción de médicos licenciados, mas no de curanderos. Por tanto, falta por conocer a través de fuentes primarias y una nueva narrativa sus intervenciones en los contextos sociales, económicos o sanitarios que se han explorado hasta el momento.

El uso de dicotomías en lo que corresponde a sus conocimientos y prácticas también se plasma en las interpretaciones. Categorías como Medicina Aborigen sin duda marcan una frontera con otros conocimientos de aquel siglo, a lo cual la Historia de la Medicina ha traído nuevas pruebas sobre lo porosa que fueron dichas fronteras. Esta última se centra en las transformaciones y circulación del conocimiento. Significa que, si se desea hacer una interpretación de los practicantes que aquí he nombrado curanderos o mojanes, es necesario preguntarse por sus itinerantes experiencias por medio de las cuales elaboraron su conocimiento.

Es probable que, a diferencia de los trabajos sobre migrantes africanos hacia América, sea más difícil encontrar registros que nos puedan mostrar la circulación del conocimiento a escala global de curanderos que practicaban la medicina en la Audiencia. Esto, a mi parecer, no resta importancia el hecho de comprender las transformaciones que dichos co- nocimientos pudieron experimentar en espacios geográficos que me parecen tan disímiles como fueron en el siglo XVIII las tierras calientes y frías. Es más, los trabajos de Gómez (2017) y Sweet (2011) son un llamado a reinterpretar los tipos de fuentes que han trabajado Salomon $(1985,1996)$ y Laviana $(1989,1996)$.

Considero que el enfoque de la experiencia permite comprender los conceptos acerca de los padecimientos de enfermedades y hasta del mismo cuerpo. Podríamos preguntarnos si la epistemología utilizada por un curandero difería en gran medida con la utilizada por un médico licenciado, o si un moján de algún pueblo podría haber aplicado de la misma forma sobre un cuerpo las hierbas medicinales que un curandero en la ciudad. ¿Si existieron límites difusos entre una epistemología y otra, pudieron materializarse en la propia concepción del cuerpo? ¿Podría la eficacia simbólica tener un papel relevante en lo que concierne al uso de ciertos objetos que lleven a experimentar enfermedades o curaciones en las personas? Me es difícil no elaborar un esquema donde lo social juzgue a un practicante por ser brujo o que las personas recurran a un curandero en la ciudad por los rumores que dicen que sí cura las enfermedades.

Finalmente, interrogo si sería más fructífero preguntarse cómo, por medio de las experiencias, diferentes actores históricos articularon y evaluaron las diversas enfermedades, curas o padecimientos en vez de centrarse en la curandería o mojanería, y en ese sentido enfocarse en artefactos culturales como lo hizo Crawford (2016). 


\section{Bibliografía}

Alchon, Suzanne. (1995). Tradiciones médicas nativas y resistencia en el Ecuador colonial. En Marcos Cuetos (Ed.), Saberes andinos: ciencia y tecnología en Bolivia, Ecuador y Perú (pp. 15-36). Lima: Instituto de Estudios Peruanos.

Alchon, Suzanne. (1996). Sociedad indígena y enfermedad en el Ecuador colonial. Quito: Abya-Yala.

Aráuz, Maritza (1999). Pueblos de indios en la costa ecuatoriana: Jipijapa y Montecristi en la segunda mitad del siglo XVIII. Guayaquil: Archivo Histórico del Guayas.

Ariza, Juan (2015). La cocina de los venenos: Aspectos de la criminalidad en el Nuevo Reino de Granada, siglos XVII y XVIII (Editorial). Bogotá.

Armus, Diego (2002). Entre médicos y curanderos. Cultura, historia y enfermedad en la América Latina moderna. Buenos Aires: Grupo Editorial Norma.

Crawford, Matthew (2016). The Andean Wonder Drug. Cinchona Bark and Imperial Science in the Spanish Atlantic, 1630 -1800. Pittsburgh: University of Pittsburgh Press.

Espinosa, Carlos (2014). Poder pastoral, acomodo y territorialidad en las Cartas Annuas jesuitas de Quito. Procesos. Revista ecuatoriana de historia, 1(38), 9-30. https://doi.org/10.29078/rp.v1i38.1

Estrella, Eduardo (1980). Medicina y Estructura Socio-Económica. Quito: Editorial Belén.

Estrella, Eduardo (1995). Ciencia ilustrada y saber popular en el conocimiento de la quina en el siglo XVIII. En M. Cuetos (Ed.), Saberes andinos: ciencia y tecnología en Bolivia, Ecuador y Perú (pp. 37-58). Lima: Instituto de Estudios Peruanos.

Estrella, Eduardo (2004). Pensamiento médico ecuatoriano I. Quito: Banco Central del Ecuador.

Few, Martha (2015). For All of Humanity. Mesoamerican and Colonial Medicine in Enlightenment Guatemala. Tucson: University of Arizona Press.

Gänger, Stefanie (2016). In their own hands: domestic medicine and 'the cure of all kinds of tertian and quartan fevers' in late-colonial Lima. Colonial Latin American Review, 25(4), 492-511. https://doi.org/10.1080/10609164.2016.1281009

Gómez, Pablo (2013). The Circulation of Bodily Knowledge in the Seventeenth-century Black Spanish Caribbean. Social History of Medicine, 26(3), 383-402. https://doi.org/10.1093/shm/hkt014

Gomez, Pablo (2017). The experiential Caribbean: creating knowledge and healing in the early modern Atlantic. Chapel Hill: University of North Carolina Press.

Hamerly, Michael (1973). Historia social y económica de la antigua provincia de Guayaquil 1763-1842. Guayaquil: Archivo Histórico del Guayas.

Laviana, María (1989). Un proceso de brujería en la costa ecuatoriana a finales del siglo XVIII: La Punta Santa Elena, 1784-1787. Anuario de estudios Americanos, (46), 93-129.

Laviana, María (1996). Brujas y curanderas de la colonia. Ecuador: Universidad Estatal de Bolívar.

Lévi-Strauss, Claude (1995). Antropología Estructural (Segunda). Buenos Aires: Paidós.

Powers, Karen (1994). Prendas con pies: migraciones indígenas y supervivencia cultural en la Audiencia de Quito. Quito: Abya - Yala.

Ramírez, Paul (2018). Enlightened Immunity. Mexico's Experiments with Disease Prevention in the Age of Reason. Stanford: Stanford University Press. 
Salomon, Frank (1985). Shamanismo y política en la última época colonial del Ecuador. Cultura, (21-b), 487-509.

Salomon, Frank (1996). La furia de Ándres Arévalo. Envoltorio de enfermedades de un shaman andino colonial. En J. Ehrenreich (Ed.), Antropología Política en el Ecuador. Perspectivas desde las culturas indígenas (pp. 115-138). Quito: Abya-Yala.

Schiebinger, Londa (2017). Secret cures of slaves: people, plants, and medicine in the eighteenth-century Atlantic world. Stanford, California: Stanford University Press.

Sweet, James (2009). Mistaken Identities? Olaudah Equiano, Domingos Álvares, and the Methodological Challenges of Studying the African Diaspora. The American Historical Review, Vol. 114, pp. 279-306. https://doi.org/10.2307/30223779

Sweet, James (2011). Domingos Álvares, African Healing, and the Intellectual History of the Atlantic World. Chapel Hill: The University of North Carolina Press.

Tapia, Amilcar (2018). De remedios y pócimas: las medicinas en el Quito del siglo 18. Revista de la Facultad de Ciencias Médicas (Quito), 43(1), 29-38. Recuperado de http://revistadigital.uce.edu.ec/index.php/CIENCIAS_MEDICAS/article/view/1454

Tardieu, Jean-Pierre (2006). El negro en la Real Audiencia de Quito (Ecuador) ss. XVI-XVIII. Quito: Abya.Yala: Instituto Francés de Estudios Andinos (IFEA).

Trouillot, Michel-Rolph (2017). Silenciando el pasado: el poder y la produccion de la Historia. Granada: Editorial Comares.

Warren, Adam (2009). Recetarios: sus autores y lectores en el Perú colonial. Histórica, 33(1), 11-41. Recuperado de http://revistas.pucp.edu.pe/index.php/historica/article/view/101

Warren, Adam (2013). From Natural History to Popular Remedy: Animals and Their Medicinal Applications among the Kallawaya in Colonial Peru. En Z. T. Martha Few (Ed.), Centering animals in Latin American History (pp. 123-148). Estados Unidos: Duke University Press. 